\title{
Further Results on the Asymptotic Agreement Problem
}

\author{
DAVID A. CASTAÑON, MEMBER, IEEE, AND DEMOSTHENIS TENEKETZIS
}

\begin{abstract}
In this paper, we develop additional results on the problem of reaching a consensus of opinion between two decision makers provided with different information. Specifically, we study the problem where the two decision makers may have different underlying probability models. We develop results characterizing the likelihood of an agreement being reached eventually in terms of the nature of the inter-decision maker communications. We also study the problem when the decision makers are aware of the possibility that they may have different models. In this case, the decision makers can reach a deadlock state where neither decision maker can learn additional information from the consensus process, and they cannot reach a consensus decision. This surprising result indicates that incorporating human uncertainty in probability assessment into the asymptotic agreement problem can lead to outcomes not anticipated in the general theory previously developed.
\end{abstract}

\section{INTRODUCTION}

$\mathrm{O}$ NE of the most important practical problems in distributed decision making is the problem of reaching a consensus of opinion among several decision makers provided with different information. This problem, called the consensus problem, consists of finding a decision, which, to each decision maker, is the correct decision according to a specific decision rule, given his information. Cne of the mechanisms suggested for reaching a consensus decision is to have decision makers exchange tentative decisions among themselves, thus exchanging part of their information. Through this process, the decision makers hope to exchange enough information in order to come to an eventual agreement on a consensus decision. The problem of asymptotic agreement is to determine conditions on this process which guarantee that a consensus decision is reached.

In [1]-[7], a Bayesian framework was developed for analyzing the problem of asymptotic agreement. Under the conditions that all decision makers share a common prior probability model, Aumann [1], Eorkar and Varaiya [2], Tsitsiklis and Athans [3], Geanakopoulos and Polemarchakis [4], and Washburn and Teneketzis [5] have shown that decision makers would approach a consensus decision under mild regularity conditions on the communication pattern. However, the condition that all decision makers share a common prior probability model restricted the applications of these results.

In subsequent papers [6], [7], Teneketzis and Varaiya showed that relaxing the condition that all decision makers share a common probability model could lead to eventual disagreement. Specifically, they showed that, when the fact that each decision maker's probability model can be different is secret knowledge [12], the consensus process can reach a state of contradiction, thereby revealing that the underlying probability models were

Manuscript received August 6, 1986; revised August 14, 1987. Paper recommended by Past Associate Editor, A. Ephremides. This work was supported by the Office of Naval Research under Contract No0014-84-C0845 .

D. A. Castañon is with Alphatech, Inc., Burlington, MA 01803

D. Teneketzis is with the Department of Electrical Engineering and Computer Science, University of Michigan, Ann Arbor, MI 48109.

IEEE Log Number 8820734. different. However, they did not extend their analysis to show what would happen once a contradiction was discovered.

In this paper, we examine in greater depth some of the issues raised by the results of Teneketzis and Varaiya [6], [7]. We limit our study to the case of two decision makers involved in the consensus problem. First, we study the question of how likely are the agreement or disagreement outcomes when the two decision makers have secret probability models. Our results establish that a contradiction outcome is likely to be reached, where the decision makers discover the existence of differences in their probability model. Then, we study the asymptotic agreement problem where each decision maker is aware of potential differences in probability models and uses an adaptive scheme to jointly identify the probability model of the other decision maker and determine his tentative decisions. Based on this assumption, we develop a new Bayesian formulation of the asymptotic agreement problem which is similar to the Bayesian formulation for games of incomplete information [8]. Using this formulation, we adapt the general framework of Washburn and Teneketzis [5] and Teneketzis and Varaiya [7] to study issues of convergence and agreement in this problem. The results of our analysis establish the existence of a new class of deadlock outcomes to the process of consensus, which is created by the ambiguities in possible probability models for each decision maker. The existence of these outcomes illustrates how differences in individual perception often result in deadlocks in bargaining situations, where progress cannot be made without the intervention of third parties.

The rest of this paper is organized as follows. In Section II, we describe the mathematical framework which is used to study the asymptotic agreement problem. In Section III, we develop additional results on the problem studied by Teneketzis and Varaiya [6], [7]. In Section IV, we discuss the asymptotic agreement problem with multiple models. Section $\mathrm{V}$ contains a discussion of the results.

\section{Problem Formulation}

Throughout this paper, we will use the following stochastic decision making model. We start with an underlying probability space, denoted by $(\Omega, \mathcal{F}, P)$. The $\sigma$-field $\mathcal{F}$ represents the set of possible events in this space. For simplicity of exposition, we assume that $\mathcal{F}$ is a finite field (all results are valid for general $\mathfrak{F}$ ). In this case, $\mathcal{F}$ can be viewed as a finite partition of $\Omega$, where the atoms of $\mathcal{F}$ correspond to the equivalence classes in this partition. In the remainder of this paper, we will refer both to finite $\sigma$-fields (partitions) and the atoms (equivalence classes) which generate them. For clarity of notation, $\sigma$-fields will be called out in script.

The stochastic model of the asymptotic agreement process starts with each decision maker (DM) receiving private information concerning the true event which occurs in $(\Omega, \mathcal{F}, P)$. This information is represented by an $\mathfrak{F}$-measurable function

$$
y^{i}: \Omega \rightarrow Y^{i}, \quad i=1,2
$$

where the superscript notation will be used throughout the paper to represent quantities which relate to DM $i$. The functions $y^{i}, i=$ 1,2 , are finite-valued and induce a partition of $\Omega$ which is coarser 
than $\mathfrak{F}$. The partition induced by $y^{i}$ is denoted as $\mathcal{Y}^{i}$. Without loss of generality, we assume that $\mathcal{F}=\mathcal{Y}^{1} \vee Y^{2}$ (the smallest $\sigma$-field which contains both $\mathcal{Y}^{1}$ and $\mathcal{Y}^{2}$ ) and that $P$ has nonzero measure on all atoms of $\mathcal{F}$.

In addition to knowledge about the true event in $(\Omega, F, P)$ described by $\mathcal{Y}^{i}$, each $\mathrm{DM}$ is assumed to have a personal probability distribution $P^{i}$ on $(\Omega, F)$ (which may be different from $P$ ), and some information concerning the personal probability distribution of the other DM. Specifically, the personal probability distributions $P^{1}$ and $P^{2}$ are assumed to be selected from a finite set $\left\{P_{i}, i \in I\right\}$ of probability distributions on $(\Omega, \mathcal{F})$, by a random lottery on $I \times I$; these distributions $P_{i}$ are assumed to be absolutely continuous with respect to $P$. The lottery is assumed by each DM to be independent of $(\Omega, \mathcal{F}, P)$. The information about the outcome of the lottery provided to DM 1 is described by subsets of $I \times I$ of the form

$$
h_{i}^{1}=\{i\} \times K_{i}
$$

where $i$ is the index of the personal probability distribution used by DM 1 , and $K_{i}$ is the set of personal probability distributions which DM 1 believes are possible for DM 2 when DM 1's probability distribution is given by $P_{i}$. Similarly, the information provided to DM 2 is described by

$$
h_{j}^{2}=K_{j} \times\{j\}
$$

where $j$ is the index of DM 2's personal probability distribution. Denote by $\mathcal{H}^{1}$ and $\mathcal{H}^{2}$ the sub- $\sigma$-fields of $2^{I} \times 2^{I}$ generated by $h^{1}, i \in I$ and $h^{2}, j \in I$, respectively.

In order to complete the probabilistic description of the information available to each DM, we must specify the subjective probabilities used by each DM to model the above lottery. Specifically, DM 1 (2) models the above lottery as the selection of a pair of indexes $(i, j) \in I \times I$ with probability $\Pi^{1}(i, j)\left(\Pi^{2}(i\right.$, $j)$ ), and assumes that this lottery is independent of $(\Omega, \mathcal{F}, P)$. Note that the subjective models of the lottery can differ between DM's.

The subjective probability models of the lottery and the outcomes of the original probability space of DM 1 (2) can be combined to form a subjective probability model on the product space $\left(\Omega \times I \times I, \mathcal{F} \times 2^{I} \times 2^{I}\right)$ with measure $Q^{1}\left(Q^{2}\right)$ defined as

$$
\begin{aligned}
& Q^{1}(f, i, j)=\Pi^{1}(i, j) P_{i}(f) \\
& Q^{2}(f, i, j)=\Pi^{2}(i, j) P_{j}(f)
\end{aligned}
$$

where $f \in \mathcal{F}$. The resulting $Q^{i}$ are probability measures on $(\Omega \times$ $\left.I \times I, \mathcal{F} \times 2^{I} \times 2^{I}\right)$. Let $\mathcal{G}$ denote the $\sigma$-field $\mathcal{F} \times 2^{I} \times 2^{I}$ on $\Omega$ $\times I \times I$.

The above formulation describes the initial information available to each DM. The class of consensus problems studied in this paper consists of selecting a decision from a set $U$ in order to minimize a jointly measurable objective function $J(\omega, u)$, where $\omega$ $\in \Omega$, and $u \in U$. We assume that $U$ is compact, and that

$$
J: \Omega \times U \rightarrow[0, \infty)
$$

is a continuous function of $u$ for each $\omega \in \Omega$. Let $g \in \mathcal{G}$ denote the available information to $\mathrm{DM} i ; g$ is an atom of the information $\sigma$-field available to DM $i$. The tentative decision of DM $i$ given information $g$ is defined by

$$
d^{i}(g) \in \underset{u \in U}{\arg \min } E_{Q^{i}}\{J(\omega, u) \mid g\}
$$

where $E_{Q} i$ denotes the expectation according to the probability distribution $Q^{i}$. Note that multiple solutions to (7) can occur. Usually, there will be a tie-breaking procedure for selecting $d^{i}(g)$. As in [5], let $\sigma\left(d^{i}(\mathcal{Q})\right)$ be the sub- $\sigma$-field of $\mathcal{G}$ generated by the decision rule $d^{i}$ when the available information $\sigma$-field is $\propto$ ( $\mathcal{\supset}$ a).
The consensus process can now be described. Each DM receives initially one measurement $y^{i}$. Based on this measurement and his personal probability model, each DM computes a tentative decision according to (7) and communicates it to the other DM. Then, each DM sequentially interprets the other DM's decision, revises his own decision due to the acquired knowledge, and communicates his new decision to the other DM. This process creates a sequence of information $\sigma$-fields $\mathcal{G}^{1}(n), \mathcal{S}^{2}(n)$. The evolution of these information fields can be described (see [5]) as a dynamical system on a lattice of sub- $\sigma$-fields of $\mathcal{G}$, with lattice operations $\vee$ and $\wedge$, where $Q \vee B$ represents the coarsest $\sigma$-field containing both $Q$ and $B$, and $Q \wedge B$ is the finest $\sigma$-field contained in both $Q$ and $B$. The relevant dynamics are:

$$
\begin{gathered}
\mathcal{S}^{1}(0)=\mathcal{Y}^{1} \times \mathcal{H}^{1} \\
\mathcal{S}^{2}(0)=\mathcal{Y}^{2} \times \mathcal{H}^{2} \\
\mathcal{S}^{1}(n+1)=\mathcal{S}^{1}(n) \vee \sigma\left(d^{2}\left(\mathcal{S}^{2}(n+1)\right)\right. \\
\mathcal{S}^{2}(n+1)=\mathcal{S}^{2}(n) \vee \sigma\left(d^{1}\left(\mathcal{S}^{1}(n)\right) .\right.
\end{gathered}
$$

Note the alternating nature of the evolution in (9). A different evolution would result if the DM's exchange information simultaneously. All of the results in this paper can be extended trivially to that case by modifying (9) appropriately.

Let $u^{1}(n)\left(u^{2}(n)\right)$ denote the value of the $n$th communication of DM 1 (DM 2), selected as a function of the information available to him according to his decision rule. For $(\omega, i, j)$ in $\Omega \times I \times I$, the DM's reach asymptotic agreement at $(\omega, i, j)$ if and only if

$$
\lim _{n \rightarrow \infty} u^{1}(n)=\lim _{n \rightarrow \infty} u^{2}(n)
$$

For $(\omega, i, j)$ in $\Omega \times I \times I$, the DM's reach a contradiction at $(\omega$, $i, j)$ if and only if there exists an $n$ and $g^{i} \in \mathcal{G}^{i}(n), i=1,2$ such that either

$$
E_{Q^{1}}\left\{I\left[g \in S: d^{2}(g)=u^{2}(n)\right] \mid g^{1}\right\}=0
$$

or

$$
E_{Q^{2}}\left\{I\left[g \in S: d^{1}(g)=u^{1}(n)\right] \mid g^{2}\right\}=0
$$

where $g^{i}$ is the atom of $g^{i}(n)$ summarizing the information obtained by DM $i$ up to time $n$. Thus, a contradiction outcome results when a communication is received for which there is no possible (e.g., nonzero probability) explanation according to the receiving DM's subjective probability model.

The above framework is an extension of the basic formulations of [1]-[7]. In [1]-[5], the common probability model formulation assumes that the set $I$ is the singleton set $\{1\}$. In this case, the probabilities $\Pi^{1}, \Pi^{2}$ are trivial, and the remaining probabilistic framework corresponds to the general framework presented in [5]. The formulation of [6] can be captured by selecting $\Pi^{1}, \Pi^{2}$ to be purely diagonal measures, of the form

$$
\Pi^{\mathrm{I}}(i, j)=0=\Pi^{2}(i, j) \quad \text { if } i \neq j
$$

and the selection of $i, j$ is such that $i \neq j$. In this case, each decision maker is convinced that the other decision maker will use the same probability model as he does. However, the initial models selected for each player are different.

\section{Genericity and Continuity of Asymptotic AgreEment: The Secret Model Problem}

In this section, we analyze the model of [6] to determine how likely are agreement or disagreement outcomes. We separate our results into two cases: the case when the decision variables are continuous, and the case when the decision variables are discrete. Throughout this section, let $i$ denote the index of DM 1 's personal 
probability model, and $j$ denote the index of DM 2's personal probability model. In order to specialize the formulation of Section II to the problems investigated in [6] and [7], we make the following assumptions. Let $A(\mathfrak{F})$ denote the atoms of $\mathfrak{F}$; then

Al: There exists some atom $g^{*} \in A(\mathcal{F})$ such that $P_{i}\left(g^{*}\right) \neq$ $P_{j}\left(g^{*}\right)$. (12)

A2: The subjective models of the lottery $\left(\Pi^{1}\right.$ and $\left.\Pi^{2}\right)$ satisfy

Assumption A1 guarantees that the differences in the DMs probability models are detectable with the available observations. Assumption A2 specifies that the knowledge that the models may be different is secret knowledge to each DM.

\section{A. Continuous Decision Variables}

When the decision space $U$ is a continuous space, we make the following additional assumption.

$A 3: U$ is a convex subset of $R^{n}$, and $J(\omega, u)$ is a strictly convex, differentiable function of $u$ for each $\omega$.

Assumption A3 guarantees that there exist unique solutions to (7). Note that $A 1-A 3$ are satisfied by the model in [6], since the decision makers exchange the conditional probability of an event $(X)$ occurring given their information. In this case,

$$
J(\omega, u)=(I\{\omega \in X\}-u)^{2}
$$

so it satisfies A3. The assumptions in [6] concerning the different probability models of the decision makers correspond to A1 and A2.

In order to characterize the likelihood of agreement or contradiction results, we need the following definitions. Let $\Pi$ be the space of all probability distributions on $(\Omega, \mathfrak{F})$. Since $\mathfrak{F}$ is a finite $\sigma$-field, $\Pi$ is a simplex in $R^{n}$, where $n$ is the cardinality of the atoms of $\mathcal{F}$. Alternatively, $\Pi$ can be viewed as a subset of $R^{n-1}$ with positive Lebesgue measure.

Definition: A result is said to be generic in $\Pi$ if and only if the set $\{\Pi \in \Pi \mid$ result is not true for $\Pi\}$ has zero $n$-1-dimensional Lebesgue measure.

Proposition 1: Let the personal probability model of DM 1 , $P_{i}$, be fixed. Under assumptions A $1-\mathrm{A} 3$, if for all $b \supset g^{*}, b \in \mathcal{G}$ [as defined after (5)], either

$$
\text { i) } u^{1}(\omega)=\underset{u \in U}{\arg \min } E_{Q^{1}}\{J(\omega, u) \mid b\} \in U^{0},
$$

or

$$
\text { ii) } u^{2}(\omega)=\underset{u \in U}{\arg \min } E_{Q^{2}}\{J(\omega, u) \mid b\} \in U^{0}
$$

where $U^{0}$ is the interior of $U$, it is generic that, for $\omega \in g^{*}$, a contradiction will be reached in the process of consensus.

Proof: Without loss of generality, assume that condition i) holds. Under assumptions $\mathrm{A} 1$ and $\mathrm{A} 2$, for $f \in \mathcal{F}$, we have

$$
\begin{aligned}
Q^{1}\left(f, i^{\prime}, j^{\prime}\right) & =0 \quad \text { if } \quad i^{\prime} \neq i \text { or } j^{\prime} \neq i \\
& =P_{i}(f) \quad \text { if } i^{\prime}=i, j^{\prime}=i \\
Q^{2}\left(f, i^{\prime}, j^{\prime}\right) & =0 \quad \text { if } i^{\prime} \neq j \text { or } j^{\prime} \neq j \\
& =P_{j}(f) \quad \text { if } i^{\prime}=j, j^{\prime}=j .
\end{aligned}
$$

In this case, the uncertain information available to each DM consists only of information about the event $\omega$ in $(\Omega, \mathfrak{F})$. Consider any instance when a tentative decision is sent from DM 1 to DM 2. Denote that decision as $u^{1}$, and the information $\sigma$-field available to player 1 about the true event in $\Omega$ as $\mathcal{F}^{1}$, where $\mathcal{F} \supset F^{\prime}$. Then, $u^{1}$ is an $\mathfrak{F}^{1}$-measurable random variable satisfying

$$
\begin{aligned}
u^{1}(\omega) & =\underset{u \in U}{\arg \min } E_{Q^{1}}\left\{J(\omega, u) \mid f^{1}(\omega)\right\} \\
& =\underset{u \in U}{\arg \min } E^{P_{i}}\left\{J(\omega, u) \mid f^{1}(\omega)\right\}
\end{aligned}
$$

where $f^{1}(\omega)$ is the atom of $\mathcal{F}^{1}$ containing $\omega ; E^{P}$ represents expectation using measures on $\mathfrak{F}$, while $E_{Q}$ represents expectation using measures on $G$. In order for a contradiction not to occur, DM 2 must be able to interpret $u^{1}(\omega)$ in terms of his own probability model; that is,

$$
u^{\prime}(\omega)=u^{21}(\omega)=\underset{u \in U}{\arg \min } E^{P_{j}}\left\{J(\omega, u) \mid f^{21}(\omega)\right\}
$$

where $f^{21}(\omega) \in F^{21}$ is DM 2's perception of the information available to DM 1 . Note that $\mathcal{F}^{21}$ is a finite field. Let $U^{21}$ denote the following subset of $U$ :

$$
\begin{aligned}
U^{21}=\{u \in U \mid u & =\arg \min E^{P_{j}}\{J(\omega, u) \mid b\} \text { for some } b \in \mathcal{F}^{21}, \\
& \text { for some } \left.\sigma \text {-field } \mathfrak{F}^{21} \text { satisfying } \mathfrak{F} \supseteq \mathfrak{F}^{21} \supseteq \mathcal{Y}^{1}\right\} .
\end{aligned}
$$

Note that $U^{21}$ is a finite set, since each $\sigma$-field $\mathcal{F}^{21}$ is finite and there are only a finite number of $\sigma$-fields satisfying the inclusion conditions. A necessary condition for the consensus process not to reach a contradiction is $u^{1}(\omega) \in I^{21}$ for all $\omega \in \Omega$. Let $\omega \in g^{*}$ in assumption $\mathrm{A} 2$; then,

$$
u^{i}(\omega)=\underset{u \in U}{\arg \min }\left\{E^{P_{i}}\left\{E^{P_{i}}(J(\omega, u) \mid \mathscr{F}) \mid f^{1}(\omega)\right\}\right\}
$$

where the inner expectation denotes the expectation with respect to the atoms $f$ of $\mathfrak{F}$ which are contained in the set $f^{1}(\omega)$. Define $J^{0}(f, u)$ for any atom $f \in A(\mathcal{F})$ as:

$$
J^{0}(f, u)=\frac{\int_{\omega \in f} J(\omega, u) P_{i}(d \omega)}{P_{i}(f)} .
$$

Then,

$$
u^{1}(\omega)=\underset{u \in U}{\arg \min }\left\{\frac{\sum_{\substack{f \subset f^{1}(\omega) \\ f \in A(\mathcal{F})}} P_{i}(f) J^{0}(f, u)}{P_{i}\left(f^{1}(\omega)\right)}\right\} .
$$

By assumption, the minimizing value is in the interior of $U$; a necessary and sufficient condition characterizing $u^{1}(\omega)$ is

$$
\sum_{\substack{f \subset f^{1}(\omega) \\ f \in A(\mp)}} P_{i}(f) \frac{\partial}{\partial u} J^{0}\left(f, u^{1}(\omega)\right)=0 .
$$

Since $u^{1}(\omega)$ must belong to $U^{21}$, this means, for some $\alpha \in U^{21}$, $g^{\prime} \in \mathscr{F}^{21}$

$$
\sum_{\substack{f \subset g^{\prime} \\ f \in A(\Im)}} P_{j}(f) \frac{\partial}{\partial u} J^{0}(f, \alpha)=0 .
$$

For each $\alpha, g^{\prime}$ the set of $P_{j}$ in $\Pi$ satisfying (14) has $n-1$ dimensional Lebesgue measure 0 , since (14) imposes a linear constraint on $P_{j}$. Since there is a finite number of $\alpha$ in $U^{21}$ and $g^{\prime}$ in $F^{21}$, the set of $P_{j}$ in II satisfying (14) for some $\alpha, g^{\prime}$ also has $n$ 1 dimensional Lebesgue measure 0 , which implies that a contradiction is generic for some $\omega$ in $g^{*}$ Q.E.D.

The results of Proposition 1 can be understood in terms of the following example.

Example 1: Let $\Omega=[0,2] \times[0,3]$. Let the $\sigma$-field $\mathcal{Y}^{\prime}$ be defined by the atoms $\left\{\omega \mid \omega \in a_{1}\right\},\left\{\omega \mid \omega \in a_{2}\right\}$, and the $\sigma$-field $\mathcal{Y}^{2}$ be defined by the atoms $\left\{\omega \mid \omega \in b_{2}\right\},\left\{\omega \mid \omega \in b_{2}\right\}$, and $\{\omega \mid \omega$ $\left.\in b_{3}\right\}$, where $a_{i}, b_{j}$ are defined in Fig. 1. Define probability models $P_{1}, P_{2}$ as:

$P_{1}(A)=\mu(A) / 6$, where $\mu$ is two-dimensional Lebesgue measure 

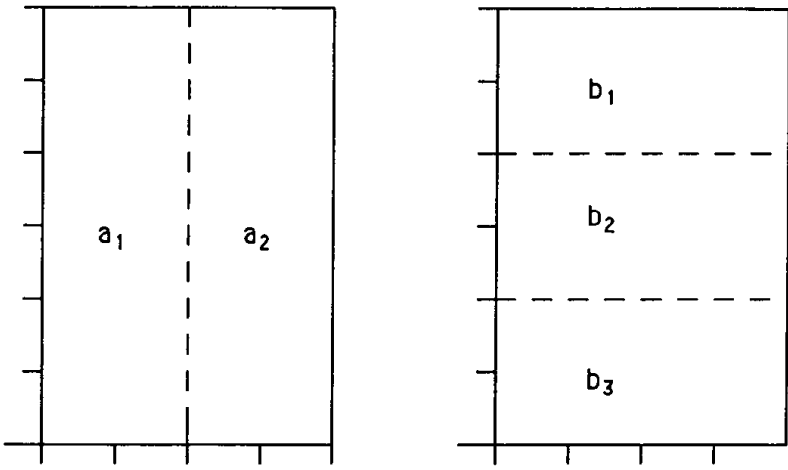

(a)

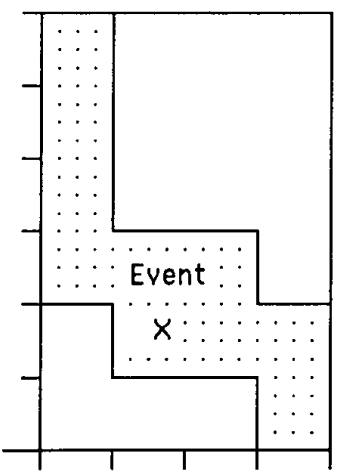

(b)

Fig. 1. (a) Information fields for each decision maker. (b) Event $X$ in the example.

$P_{2}(A)=\mu\left(A \cap b_{1}\right) / 36+\mu\left(A \cap b_{2}\right) / 24+7 \mu\left(A \cap b_{3}\right) / 72$

$P_{1}$ is uniformly distributed over $\Omega$, while $P_{2}$ is uniformly distributed conditioned on $b_{i}$, but has

$$
P_{2}\left(b_{1}\right)=1 / 6 ; P_{2}\left(b_{2}\right)=1 / 4 ; P_{2}\left(b_{3}\right)=7 / 12 .
$$

Let $i=1$, while $j=2$, so that DM 1 uses probability model $P_{1}$, while DM 2 uses probability model $P_{2}$. The decision rule used by the DM's is defined by (13), where the event is event $X$ in Fig. 1 .

As noted in [6], when $\omega \in a_{1} \cap b_{3}$, and DM 1 communicates first, there is eventual agreement, although the DM's have very different reasons for reaching that agreement. In order to illustrate that disagreement is generic, we will show that arbitrarily small perturbations to $P_{2}$ result in disagreement. Specifically, let

$P_{2}\left(b_{1}\right)=\left(2+\epsilon_{1}\right) / 12 ; P_{2}\left(b_{2}\right)=\left(3+\epsilon_{2}\right) / 12 ;$

$$
P_{2}\left(b_{3}\right)=\left(7-\epsilon_{1}-\epsilon_{2}\right) / 12 .
$$

As in [6], DM 1's first communication is $u^{1}=0.5$. In order for this value not to be a contradiction, either

$$
3 \epsilon_{1}+2 \epsilon_{2}=0
$$

or

$$
\epsilon_{1}+2 \epsilon_{2}=4
$$

The two-dimensional Lebesgue measure of the set of all $\epsilon_{1}, \epsilon_{2}$ satisfying (15) or (16) is 0 , since it is the union of two lines. Hence, for almost all choices of $\epsilon_{1}, \epsilon_{2}$, a contradiction will be reached in the first communication.

The reason for the genericity of disagreement in Proposition is that, although each DM can observe only a finite number of observation values, he can communicate a continuous number of decisions. This enables the other DM to detect differences in the probability models. Conditions i) or ii) in Proposition 1 guarantee that the announced decisions will vary with small differences in probability models.

\section{B. Discrete Decision Variables}

In this subsection, we assume that the space $U$ is discrete. Let $f$ denote an atom of $\mathcal{F}$. We define a metric on $\Pi$ as follows.

For $P_{1}, P_{2} \in \Pi$,

$$
d\left(P_{1}, P_{2}\right)=\max _{f \in F}\left|P_{1}(f)-P_{2}(f)\right| .
$$

This metric is equivalent to the Euclidean metric on II.

Definition: An agreement or disagreement result is said to be continuous in $\Pi$ at $\boldsymbol{P}_{1}, \boldsymbol{P}_{2}$ if agreement or disagreement continues to hold for all $P_{1}{ }^{\prime}, P_{2}{ }^{\prime}$ in a neighborhood of $P_{1}, P_{2}$.

Assume the following.

A4: For any $f \in \mathcal{F}$, there exists unique $u^{1}, u^{2}$ in $U$ such that

$$
\begin{aligned}
u^{1} & =\underset{u \in U}{\arg \min } E_{Q^{1}}\{J(\omega, u) \mid \omega \in f\} \\
& =\underset{u \in U}{\arg \min } E^{P_{i}}\{J(\omega, u) \mid \omega \in f\} \\
u^{2} & =\underset{u \in U}{\arg \min } E_{Q^{2}}\{J(\omega, u) \mid \omega \in f\} \\
& =\underset{u \in U}{\operatorname{argmin}} E^{P_{j}}\{J(\omega, u) \mid \omega \in g\} .
\end{aligned}
$$

With this assumption, we have the following characterization of agreement or disagreement outcomes.

Proposition 2: Under assumptions A1, A2, and A4, if agreement occurs for $P_{i}, P_{j}$, it is continuous in $\Pi$. If disagreement occurs for $P_{i}, P_{j}$, it is also continuous in $\Pi$.

Proof: Without loss of generality, assume that $P_{i}, P_{j}$ result in agreement. Let $\mathcal{F}^{1}(n)\left(\mathcal{F}^{2}(n)\right)$ DM 1's (DM 2's) sequence of sub- $\sigma$-fields of $\mathcal{F}$ generated in the consensus process according to $(8),(9)$. For any time interval $n$, the atoms of $\mathcal{F}^{-1}(n)$ and $\mathfrak{F}^{2}(n)$ are elements of $\mathcal{F}$. Let $f$ denote an atom of $\mathcal{F}$. For any $g \in \mathcal{F}$, define the function



This is a continuous function in $\Pi$. Because of assumption A4, we can find a $\delta^{1}(g)$ such that, for $d\left(P_{i}, P_{1}\right) \leq \delta^{1}(g)$

$$
\underset{u \in U}{\arg \min } L\left(P_{i}, g, u\right)=\underset{u \in U}{\arg \min } L\left(P_{1}, g, u\right) .
$$

A similar result can be established in terms of $\delta^{2}(A)$ for DM 2's decisions. Select $\delta$ as

$$
\delta=\min _{g \in \mathscr{F}}\left\{\delta^{1}(g), \delta^{2}(g)\right\} .
$$

This minimum exists because there are only a finite number of $g$ in $\mathcal{F}$. This choice of $\delta$ guarantees that the exchanged sequence of decisions and the $\sigma$-fields inferred by the other DM are the same for all probabilities $P_{1}, P_{2}$ satisfying

$$
\begin{aligned}
& d\left(P_{i}, P_{1}\right) \leq \delta \\
& d\left(P_{j}, P_{2}\right) \leq \delta,
\end{aligned}
$$

thereby completing the proof.

Q.E.D.

The result of Proposition 2 depends critically on assumption 
A4. However, an argument similar to the proof of Proposition 1 establishes the following result.

Proposition 3: If, for each atom $f \in \mathfrak{F}$,

$$
J^{0}(f, u) \neq J^{0}(f, v) \quad \text { if } u \neq v,
$$

then assumption $\mathrm{A} 4$ is generic in $\Pi$.

Proof: Let $f$ denote the atoms of $\mathcal{F}$. If assumption $\mathrm{A} 4$ does not hold for $P_{i}$, there must exist a set $g \in \mathfrak{F}$ and $u, v \in U, u \neq v$, such that

$$
E^{P_{i}}\{J(\omega, u) \mid \omega \in g\}=E^{P_{i}}\{J(\omega, v) \mid \omega \in g\} .
$$

This implies that

$$
\sum_{\substack{f=g \\ f \in \mathcal{A}(\mathfrak{F})}} P_{i}(f) J^{0}(f, u)=\sum_{\substack{f \subset g \\ f \in A(\mathcal{F})}} P_{i}(f) J^{0}(f, v) .
$$

Since $J^{0}(f, v) \neq J^{0}(f, u)$ for any atom of $\mathcal{F}$, this implies that the set of $P_{i}$ which satisfy this equation has Lebesgue measure 0 in $\Pi$. A similar argument for $P_{j}$ completes the proof.

Q.E.D.

Example 2: Let $(\Omega, \mathfrak{F}), \mathcal{Y}^{1}, \mathcal{Y}^{2}$, and $X$ be defined as in Example 1. Define probability models $P_{3}, P_{4}$ as

$$
P_{3}(A)=2 \mu\left(A \cap a_{1}\right) / 15+\mu\left(A \cap a_{2}\right) / 5
$$

where $\mu$ is two-dimensional Lebesque measure,

$$
\begin{aligned}
P_{4}(A)= & \mu\left(A \cap a_{2}\right) / 5+4 \mu\left(A \cap a_{1} \cap b_{1} \cap X\right) / 95 \\
& +12 \mu\left(A \cap a_{1} \cap b_{1} \cap X^{c}\right) / 95+12 \mu\left(A \cap b_{2} \cap a_{1}\right) / 95 \\
& +36 \mu\left(A \cap a_{1} \cap b_{3} \cap X\right) / 95 \\
& +12 \mu\left(A \cap a_{1} \cap b_{3} \cap X^{c}\right) / 95
\end{aligned}
$$

where $X^{c}$ is the complement of $X$ in $\Omega$. Rather than work with the unconditional probabilities, what is important is to evaluate the conditional probabilities of events given available information. Thus, $P_{3}$ is uniformly distributed conditioned on $a_{i}$, with

$$
P_{3}\left(a_{1}\right)=0.4 ; P_{3}\left(a_{2}\right)=0.6 \text {. }
$$

$P_{4}$ has the same distribution as $P_{3}$ on $a_{2}$, but differs on $a_{1}$, as

$$
\begin{gathered}
P_{4}\left(X \mid a_{1} \cap b_{1}\right)=0.25 ; P_{3}\left(X \mid a_{1} \cap b_{1}\right)=0.5 \\
P_{4}\left(X \mid a_{1} \cap b_{2}\right)=P_{3}\left(X \mid a_{1} \cap b_{2}\right)=0.75 \\
P_{4}\left(X \mid a_{1} \cap b_{3}\right)=0.50 ; P_{3}\left(X \mid a_{1} \cap b_{3}\right)=0.25 \\
P_{4}\left(X \mid a_{1} \cap\left(b_{2} \cup b_{3}\right)\right)=0.6 ; P_{3}\left(X \mid a_{1} \cap\left(b_{2} \cup b_{3}\right)\right)=0.5
\end{gathered}
$$

$$
P_{4}\left(X \mid a_{1}\right)=10 / 19 ; P_{3}\left(X \mid a_{1}\right)=0.5 .
$$

Let $U=\{0,1\}$. Let

$$
\begin{aligned}
J(\omega, 0)=0.53 & & \text { if } \omega \in X, \\
=0 & & \text { if } \omega \in X^{c}, \\
J(\omega, 1)=0 & & \text { if } \omega \in X, \\
=0.47 & & \text { if } \omega \in X^{c} .
\end{aligned}
$$

With this definition of $J$, the optimal decision for DM 1 given an information set $g \in \mathcal{F}$ is given by

$$
\begin{aligned}
u^{1} & =1 \text { if } P_{i}(\omega \in X \mid g)>0.47 \\
& =0 \text { otherwise. }
\end{aligned}
$$

The same decision rule is optimal for DM 2, using the probability $P_{j}$.

Assume $i=3$, while $j=4$, that DM 1 exchanges his decision first, and that the DM's alternate in exchanging decisions. As in Example 1, assume that $\omega \in a_{1} \cap b_{3}$. From (18e) and (19), DM 1 's initial decision is 1 . That is, $u^{1}(1)=1$. If DM l's information had been $a_{2}$, his decision would have been $u^{1}=0$. Hence, according to DM 1 , he has signaled $\omega \in a_{1}$ to DM 2 . According to DM 2's probability model, $u^{1}(1)=1$ implies $\omega \in a_{1}$. Hence, DM 2 believes $\omega \in a_{1} \cap b_{3}$. His optimal decision is $u^{2}(1)=1$, because of $(18 \mathrm{c})$. According to DM 2 , his decision has signaled $\omega$ $\in b_{2} \cup b_{3}$, because of (18a) and (18b).

Because of (18a)-(18c), DM 1 interprets $u^{2}(1)=1$ to mean $\omega$ $\in a_{1} \cap\left(b_{1} \cup b_{2}\right)$. His optimal decision is $u^{1}(2)=1$. Hence, a consensus has been reached at $u^{1}(2)=u^{2}(2)=1$. However, they have reached this agreement for the wrong reasons, since DM 1 believes $\omega \in a_{1} \cap\left(b_{1} \cup b_{2}\right)$, whereas in actuality, $\omega \in a_{1} \cap b_{3}$ ! Note that any changes in either $P_{3}$ or $P_{4}$ which would change the numbers in (18a)-(18e) by less than 0.02 would continue to result in agreement.

Suppose that the order of communication is reversed, so that DM 2 communicates first. The optimal decision $u^{2}(1)=1$. Note that $P_{4}\left(X \mid \omega \in b_{2}\right)<0.45, P_{4}\left(X \mid \omega \in b_{1}\right)<0.2$. Hence, DM 2 believes he has signaled $\omega \in b_{3}$. According to DM 1 , he interprets $u^{2}(1)=1$ to mean $\omega \in b_{3}$. Hence, he believes $\omega \in a_{1}$ $\cap b_{3}$. His optimal decision, according to $(18 \mathrm{c})$, is $u^{1}(1)=0$. This decision cannot be understood by DM 2 , because he expected $u^{1}(1)=1$ whether DM 1 knew $a_{1}$ or $a_{2}$. Hence, the DM's have reached a contradiction. Note that this contradiction will be reached even if $P_{3}$ or $P_{4}$ are modified by 0.02 . Hence, the disagreement outcome is also continuous.

The above results illustrate that, when the decision spaces are discrete, small discrepancies in the probability models of the decision makers will not affect the consensus process. They also show that the set of pairs of probability models for which consensus accurs has positive Lebesgue measure in $\Pi$, unlike the result in the continuous decision case of the previous subsection. However, the set of pairs of probability models for which contradictions occur also has positive Lebesgue measure. Hence, contradictions are common phenomena in the consensus process.

The question still remains: How does the consensus process proceed once a contradiction is encountered? Such a contradiction reveals that the basic assumption that $\Pi^{1}(i, j)=0$ (or $\Pi^{2}(i, j)=$ $0)$ if $i \neq j$, is violated. In the next section, we present a plausible model for the continuation of the consensus process, and study its implications.

\section{Asymptotic Agreement with Multiple Probability MODELS}

When the decision makers in the consensus process have different subjective views of the world, and these differences are secret knowledge, the results of the previous section and [6], [7] show that a contradiction outcome is often reached whereby the existence of these differences becomes common knowledge. At this point, our model of how the consensus process proceeds is that each decision maker models statistically the types of probability models which the other decision maker may be employing, and acquires information through the consensus process concerning the possible models used by the other DM, and the uncertainty in the event spaci $\Omega$. Within this framework, we investigate convergence and agreement issues for two cases: when the statistics of the types of probability models are common knowledge, and when these statistics are secret knowledge. The analysis is based on the mathematical formulation developed in Section II where the probability distributions $\Pi^{1}, \Pi^{2}$ represent the statistics used by each DM.

Initially, we will make the following assumption.

A5: $\Pi^{1}, \Pi^{2}$ are common knowledge.

Recall that $\Pi^{1}, \Pi^{2}$ are the subjective probabilities on the space 
of possible model pairs $(i, j)$. Assumption A5 implies that the subjective statistical distribution of possible probability models for each decision maker is known to the other decision maker, and this fact is common information. Note that we do not assume that these distributions are equal. This allows DM 1 to believe he has a different range of possible decision models than DM 2 has, and vice versa.

The decision rules $d^{i}$ used by the DM's are described by (7). A decision rule $d$ is said to satisfy the agreement condition if, for $\sigma$ fields $S_{1}, S_{2}$ such that $S_{1} \supseteq G_{2} \supseteq \sigma\left(d\left(S_{1}\right)\right)$, then $d\left(S_{1}\right)=d\left(S_{2}\right)$. In [5], it was shown that such a rule satisfies the agreement condition. The agreement condition implies that, if a decision is based on information which is common to the information $\sigma$-fields $S_{2}$ and $S_{1}$, then knowledge of either $S_{2}$ or $S_{1}$ would result in the same decision.

Proposition 4: Under Assumptions A5 and A6, if $i=j$ and $\omega$ $\in \Omega$ is such that the consensus process reveals that $i=j$ to both decision makers, then the decision makers reach a consensus for $\omega$.

Proof: Without loss of generality, let $i=j=1$. As in (8) and (9) let $\mathcal{G}^{1}(n)\left(\mathcal{S}^{2}(n)\right)$ denote the information $\sigma$-field available to DM 1 (2) at time $n$. Because of A5, the evolutions indicated in (8), (9) are common knowledge. Note that these dynamical systems are evolving on a lattice of finite fields, and that they generate a strictly increasing sequence of $\sigma$-fields. Hence, after some finite time $t$, a limit must be reached such that, for all $s>t$,

$$
\begin{aligned}
& \mathcal{G}^{1}(s)=\mathcal{G}^{1}=\mathcal{G}^{1} \vee \sigma\left(d^{2}\left(\mathcal{G}^{2}\right)\right) \\
& \mathcal{S}^{2}(s)=\mathcal{G}^{2}=\mathcal{S}^{2} \vee \sigma\left(d^{1}\left(\mathcal{G}^{1}\right)\right) .
\end{aligned}
$$

Equations (20) establish that the consensus process converges to a limit; that is,

$$
\lim _{n \rightarrow \infty} u^{1}(n)=u^{1 *} ; \lim _{n \rightarrow \infty} u^{2}(n)=u^{2 *}
$$

In addition, (20) imply that

$$
\begin{aligned}
& \mathcal{S}^{1} \supseteq \sigma\left(d^{2}\left(\mathcal{G}^{2}\right)\right) \\
& \mathcal{S}^{2} \supseteq \sigma\left(d^{1}\left(\mathcal{S}^{1}\right)\right) .
\end{aligned}
$$

Furthermore, the fields generated by a decision rule are contained in the information available for decisions. That is,

$$
\begin{aligned}
& \mathcal{G}^{2} \supseteq \sigma\left(d^{2}\left(\mathcal{G}^{2}\right)\right) \\
& \mathcal{G}^{1} \supseteq \sigma\left(d^{1}\left(\mathcal{G}^{1}\right)\right) .
\end{aligned}
$$

Hence,

$$
\begin{aligned}
& \mathcal{G}^{2} \supseteq \mathcal{G}^{2} \wedge \mathcal{G}^{1} \supseteq \sigma\left(d^{2}\left(\mathcal{G}^{2}\right)\right) \\
& \mathcal{G}^{1} \supseteq \mathcal{G}^{2} \wedge \mathcal{G}^{1} \supseteq \sigma\left(d^{1}\left(\mathcal{G}^{1}\right)\right) .
\end{aligned}
$$

By assumption, the decision rules satisfy the agreement condition. Hence, by (24),

$$
\begin{aligned}
& d^{1}\left(\mathcal{G}^{1}\right)=d^{1}\left(\mathcal{G}^{1} \wedge \mathcal{G}^{2}\right) \\
& d^{2}\left(\mathcal{G}^{2}\right)=d^{2}\left(\mathcal{G}^{1} \wedge \mathcal{G}^{2}\right) .
\end{aligned}
$$

Select $\omega \in \Omega$ such that $i=1=j$ is common knowledge. Since the $\sigma$-fields are increasing, there is an atom $g$ containing $\omega$ in $\mathcal{G}^{1}$ $\wedge \mathcal{G}^{2}$ of the form $g=(A, 1,1)$, where $\Omega \supseteq A$. Since $g$ is an element of both limiting fields $\mathcal{S}^{1}$ and $\mathcal{G}^{2}$, it follows that

$$
\begin{aligned}
d^{1}(g) & =\underset{u \in U}{\arg \min } E_{Q^{1}}\{J(\omega, u) \mid g\} \\
& =\underset{u \in U}{\arg \min }\left\{\Pi^{1}(1,1) E^{P_{1}}\{J(\omega, u) \mid \omega \in A\}\right\} .
\end{aligned}
$$

Similarly, since $j=1$ in $g$,

$$
\begin{aligned}
d^{2}(g) & =\underset{u \in U}{\arg \min } E_{Q^{2}}\{J(\omega, u) \mid g\} \\
& =\underset{u \in U}{\arg \min }\left\{\Pi^{2}(1,1) E^{P_{1}}\{J(\omega, u) \mid \omega \in A\}\right\}
\end{aligned}
$$

where $E^{P}{ }_{i}$ is used to denote expectation with respect to the measure $P_{i}$ on $(\Omega, \mathfrak{F})$. The functions being minimized are simple multiples of each other. Thus, $d^{2}(g)=d^{1}(g)$ for all such $g$. Coupled with (25), this completes the proof.

According to the above proposition, even if the decision makers have the same probability model, consensus is not guaranteed unless it becomes common knowledge that $i=j$. On the other hand, the condition that $\omega \in \Omega$ is such that the consensus process reveals that $i=j$ is sufficient but not necessary for reaching a consensus. These points are illustrated by the following example.

Example 3: Let $(\Omega, \mathfrak{F}), \mathcal{Y}^{1}, \mathcal{Y}^{2}, X, P_{3}, P_{4}$, and $U$ be defined as in Example 2. Consider the decision rule defined in (7). Let

$$
\begin{aligned}
J(\omega, 0)=0.57 & & \text { if } \omega \in X, \\
=0 & & \text { if } \omega \in X^{c}, \\
J(\omega, 1)=0 & & \text { if } \omega \in X, \\
=0.43 & & \text { if } \omega \in X^{c} .
\end{aligned}
$$

With this definition of $J$, the optimal decision for DM 1 given an information set $A$ is given by

$$
\begin{aligned}
u^{1} & =1 \text { if } P_{i}(\omega \in X \mid A)>0.43 \\
& =0 \text { otherwise. }
\end{aligned}
$$

Assume that $i=j=3$, so that both decision makers have the same probability model. Assume further that there exists a probability distribution $R$ on $\{3,4\}$ such that

$$
\Pi^{1}(i=m, j=k)=\Pi^{2}(i=m, j=k)=R(m) R(k) .
$$

That is, each decision maker believes that the other DM's decision model is selected independently from a known statistical population, where the set of possible models was $\left\{P_{3}, P_{4}\right\}$. Furthermore, the statistics of the selection are known identically to both decision makers. However, the precise model selected is private knowledge provided to each decision maker.

Assume $R(3)=0.1$. As in Example 2, we assume that the DM's alternate exchanging tentative decisions. As in Example 2, DM 1's first decision is $u^{1}(1)=1$, and this signals that $\omega \in a_{1}$ to DM 2. This decision does not reveal whether $i=3$ or $i=4$, because if $i=3$ or 4 , the same decision would be made. At this point in the consensus process, DM 2 knows that $\omega \in a_{1} \cap b_{3}$ whether $i=3$ or 4 . Hence, by (7), since $j=3$, the optimal decision is $u^{2}(1)=0$. In order to identify the information signaled by this decision, we must examine the optimal decisions corresponding to the possible information sets that DM 2 could have, from DM 1's perspective. These decisions are:

$$
\begin{aligned}
& \text { if } j=3, \omega \in a_{1} \cap b_{1} \text {, then } u^{2}(1)=1 \text { by (18a) } \\
& \text { if } j=3, \omega \in a_{1} \cap b_{2} \text {, then } u^{2}(1)=1 \text { by (18b) } \\
& \text { if } j=3, \omega \in a_{1} \cap b_{3} \text {, then } u^{2}(1)=0 \text { by (18c) } \\
& \text { if } j=4, \omega \in a_{1} \cap b_{1} \text {, then } u^{2}(1)=0 \text { by (18a) } \\
& \text { if } j=4, \omega \in a_{1} \cap b_{2} \text {, then } u^{2}(1)=1 \text { by (18b) } \\
& \text { if } j=4, \omega \in a_{1} \cap b_{3} \text {, then } u^{2}(1)=1 \text { by (18c). }
\end{aligned}
$$

Hence, DM 1 knows that either $j=3, \omega \in a_{1} \cap b_{3}$ or $j=4, \omega$ 
$\in a_{1} \cap b_{1}$. According to (7), his optimal decision is selected as

$$
\begin{aligned}
u^{1}(2)= & \underset{u \in U}{\arg \min } E_{Q^{1}}\{J(\omega, u) \mid F\} \\
= & \underset{u \in\{0,1\}}{\arg \min }\{R(3)[(0.25)(0.53) I\{u=0\} \\
& +(0.75)(0.47) I\{u=1\}]+R(4)[(0.5)(0.53) I\{u=0\} \\
& +(0.5)(0.47) I\{u=1\}]\}
\end{aligned}
$$

where $I\{\}$ is the indicator function, and a constant scaling factor has been omitted. It is easy to see that, for $R(3)<0.12, u^{1}(2)=$ 1. Note that, if $i=4,(28)$ becomes

$$
\begin{aligned}
u^{1}(2)= & \underset{u \in\{0,1\}}{\arg \min }\{R(4)[(2 / 3)(0.53) I\{u=0\} \\
& +(2)(0.47) I\{u=1\}]+R(3)[(3)(0.53) I\{u=0\} \\
& +(3)(0.47) I\{u=1\}]\} .
\end{aligned}
$$

So, $u^{1}(2)$ should be 0 if $i=4$. Therefore, $u^{1}(2)=1$ signals that $i$ $=3$ to DM 2 .

DM 2 now knows $i=j=3$, and $\omega \in a_{1} \cap b_{3}$. As before, his optimal decision is $u^{2}(2)=0$. This decision does not convey any additional information to DM 1 , because the decision $u^{2}(2)=0$ did not depend on the information $i=3$. Since DM 1 obtains no additional information, his tentative decision continues to be $u^{1}(2)$ $=1$, and the two decision makers agree that an agreement cannot be reached. The common information which forms the basis for this disagreement can be summarized in the atom of $\mathcal{F} \times 2^{I} \times s^{I}$ given by

$\left\{\left(\omega, i_{1}, i_{2}\right) \mid\left(\omega \in a_{1} \cap b_{3}, i_{1}=i_{2}=3\right)\right.$,

$$
\text { or } \left.\left(\omega \in a_{1} \cap b_{1}, i_{1}=3, i_{2}=4\right)\right\} \text {. }
$$

Consider now the same problem, but assume that DM 2 communicates first. Then, since $\omega \in b_{3}$ and $P_{3}\left(X \mid b_{3}\right)=0.55$, then $u^{2}(1)=1$.DM 1 observes $a_{1}$ and receives $u^{2}(1)=1$; by the same argument as above, he concludes that DM 2 has observed either $b_{2}$ or $b_{3}$ and uses either model 3 or 4 . His decision is $u^{1}(1)$ $=1$. When DM 2 receives $u^{1}(1)=1$, he concludes that $\omega \in b_{3}$ $\cap\left(a_{1} \cup a_{2}\right)$ and DM 1 uses either model 3 or 4 . Thus, DM 1's decision did not convey any additional information to DM 2 . Thus, $u^{2}(2)=1$, which does not convey any information to DM 1 . Hence, $u^{1}(2)=1$ and the DM's agree, even though the event $\omega$ is such that the consensus process does not reveal that the DM's have the same model.

The results of Example 3 are rather surprising. Unlike the cases studied in [6] or [7], there is no unmodeled secret information present in this consensus process. Indeed, both DM's are actually using the same probability model; furthermore, they have identical probability distributions over the class of probability models, and this is common knowledge! Nevertheless, a disagreement outcome occurs due to deadlock. This implies that even admitting the possibility that the other DM can have a different subjective probability model than your own is sufficient to prevent reaching a consensus. The reason for this effect is the difference in the probability distributions used by each DM in (7) when one DM is unable to identify the probability model used by the other DM.

How likely is it that the conditions of Proposition 4 are met? Our analysis of the previous section can be extended to establish the following propositions.

Proposition 5: Assume that the decision space $U$ is continuous, and that Assumption $\mathrm{A} 3$ is true. Assume $i=j$. Assume additionally that, for all $g \in \mathcal{S}$ such that $\omega \in g$,

$$
\begin{aligned}
& \text { i) } u^{1}(\omega)=\underset{u \in U}{\arg \min } E_{Q^{1}}\{J(\omega, u) \mid g\} \in U^{0} \\
& \text { ii) } u^{2}(\omega)=\underset{u \in U}{\arg \min } E_{Q^{2}}\{J(\omega, u) \mid g\} \in U^{0}
\end{aligned}
$$

where $U^{0}$ is the interior of $U$. Then, the outcome that the consensus process will reveal that $i=j$ is generic in $\Pi^{k}$, where $k$ is the cardinality of $I$.

Proposition 6: If the decision space $U$ is discrete, $i=j$, and for any $g \in G$ there exists unique $u^{1}, u^{2}$ in $U$ such that

$$
\begin{aligned}
& u^{1}=\underset{u \in U}{\arg \min } E_{Q^{1}}\{J(\omega, u) \mid g\} \\
& u^{2}=\underset{u \in U}{\arg \min } E_{Q^{2}}\{J(\omega, u) \mid g\}
\end{aligned}
$$

then the outcome that a consensus process reveals that $i=j$ for a specific $\omega$ is continuous in $\Pi^{k}$.

The proof of these propositions follows directly the proof of Propositions 1 and 2, and will not be reproduced here. Essentially, Proposition 5 is based on the fact that the set of probability models for which a continuous decision fails to discriminate among a finite set of models has zero Lebesgue measure in the space of all possible probability models. Under the assumptions of Proposition 6 , one can show that the sequence of $\sigma$-fields generated in the consensus process does not change with small perturbations in the set of individual probability models.

When $i \neq j$, it is possible to show, by arguments similar to those leading to (19)-(24) that the sequence of decisions $\left\{d^{1}\left(S^{1}(n)\right)\right\}$ and $\left\{d^{2}\left(S^{2}(n)\right)\right\}$ will converge to $d^{1}\left(G^{1}\right)$ and $d^{2}\left(G^{2}\right)$, respectively. However, since the probabilistic models of DM 1 and DM 2 are not the same, whether or not a consensus is reached depends on the event $\omega \in \Omega$ and the order of communication. If $g$ is an atom of $S^{1} \wedge S^{2}$ containing $\omega \in \Omega$, then a consensus will be reached if $d^{1}(g)=d^{2}(g)$.

The above results were based on assumption $\mathrm{A} 5$ that the underlying statistical models $\Pi^{1}$ and $\Pi^{2}$ used by each $D M$ are common knowledge. When these models are different, and this fact is secret knowledge, and the decision processes of DM 1 and DM 2 are consistent with their own beliefs, then the consensus process reaches one of three different outcomes after a finite number of communications:

1) A consensus is reached,

2) DM 1 and DM 2 realize that their underlying statistical models are inconsistent,

3) DM 1 and DM 2 agree to disagree because they cannot gather any additional information from the consensus process.

In order to establish this, we must describe the evolution of the decision processes according to each DM's subjective decision model, and determine what each DM's model predicts. Then, we compare the predicted communications to the actual communications heard. Let $u^{11}(n), u^{12}(n)$ denote the decisions of DM 1 and DM 2 at stage $n$ according to DM 1's subjective decision model. Similarly, let $u^{21}(n), u^{22}(n)$ denote the decisions of DM 1 and DM 2 at stage $n$ according to DM 2's subjective decision model. Then, according to DM l's view,

$$
\begin{aligned}
& u^{11}(n)=d^{11}\left(y^{1}, u^{12}(1), \cdots, u^{12}(n-1)\right) \\
& u^{12}(n)=d^{12}\left(y^{2}, u^{11}(1), \cdots, u^{11}(n-1)\right)
\end{aligned}
$$

where $d^{\text {tj }}$ denotes the decisions formed by the decision rule $d$ according to the probability measure $Q^{1}$. Similarly, according to DM 2's view,

$$
\begin{aligned}
& u^{21}(n)=d^{21}\left(y^{1}, u^{12}(1), \cdots, u^{12}(n-1)\right) \\
& u^{22}(n)=d^{22}\left(y^{2}, u^{11}(1), \cdots, u^{11}(n-1)\right)
\end{aligned}
$$

where $d^{2 j}$ denotes the decisions formed by the decision rule $d$ according to the probability measure $Q^{2}$. Equations (29) and (30) describe the evolution of the consensus process according to DM 1 's and DM 2's perception, respectively.

To determine what DM 1 and DM 2 predict about the outcome of the decision processes in terms of their own perceptions, we define four sequences of information $\sigma$-fields, representing DM 
1's actual knowledge $\left(g^{11}(n)\right)$, DM 1's belief of DM 2's knowledge $\left(\mathcal{G}^{12}(n)\right)$, DM 2 's actual knowledge $\left(\mathcal{G}^{22}(n)\right)$, and DM 2 's belief of DM 1's knowledge $\left(S^{21}(n)\right)$. These fields evolve under communications as

$$
\begin{aligned}
& \mathcal{G}^{11}(n+1)=\mathcal{G}^{11}(n) \vee \sigma\left(d^{12}\left(\mathcal{G}^{12}(n)\right)\right) \\
& \mathcal{G}^{12}(n+1)=\mathcal{G}^{12}(n) \vee \sigma\left(d^{11}\left(\mathcal{S}^{11}(n)\right)\right) \\
& \mathcal{S}^{21}(n+1)=\mathcal{G}^{21}(n) \vee \sigma\left(d^{22}\left(\mathcal{G}^{22}(n)\right)\right) \\
& \mathcal{S}^{22}(n+1)=\mathcal{G}^{22}(n) \vee \sigma\left(d^{21}\left(\mathcal{S}^{21}(n)\right)\right)
\end{aligned}
$$

with initial conditions

$$
\begin{aligned}
& \mathcal{S}^{11}(0)=\mathcal{G}^{21}(0)=\mathcal{Y}^{1} \times \mathcal{K}^{1} \\
& \mathcal{S}^{22}(0)=\mathcal{S}^{12}(0)=\mathcal{Y}^{2} \times \mathcal{H}^{2} .
\end{aligned}
$$

As before, these evolutions occur in a lattice of $\sigma$-fields where the maximal element is a finite $\sigma$-field. Hence, repeating the logic of the proof of Proposition 4 establishes that the consensus process will reach steady state after a finite number of iterations. Denote this finite number as $T$.

To establish the type of outcomes possible, we must examine the consensus process closely. At stage 1, DM 1's decision is $u^{11}(1)=d^{11}\left(y^{1}\right)$. This message is transmitted to DM 2, who must interpret this message according to his own subjective decision model. That is, he must find realizations $\left(i^{\prime}, y^{1^{\prime}}\right)$ of possible models and observation values such that $u^{11}(1)=d^{21}\left(y^{1 \prime}\right)$. For a consistent interpretation, one must have

$$
P_{j}\left\{\left(y^{1 \prime} \mid u^{11}(1)=d^{21}\left(y^{1 \prime}\right), i=i^{\prime}\right\}>0 \quad \text { for some } i^{\prime} \in I .\right.
$$

If this is not possible, DM 2 will discover that the decision models are inconsistent, leading to outcome 2. Otherwise, DM 2 selects $u^{22}(1)=d^{22}\left(y^{2}, u^{11}(1)\right)$.

At this stage, DM 1 must interpret consistently the communications heard from DM 2. As before, he must find realizations $\left(j^{\prime}\right.$, $y^{2 \prime}$ ) of possible models and observation values such that $u^{22}(1)=$ $d^{12}\left(y^{2}, u^{11}(1)\right)$. For a consistent interpretation, one must have

$P_{i}\left\{\left(y^{2 \prime} \mid u^{22}(1)=d^{12}\left(y^{2 \prime}, u^{11}(1)\right), j=j^{\prime}\right\}>0\right.$

for some $j^{\prime} \in I$.

Define $P^{1}\left(n, i^{\prime}\right), P^{2}\left(n, j^{\prime}\right)$ as follows:

$$
\begin{aligned}
P^{1}\left(n, i^{\prime}\right) & =P_{j}\left\{\left(y^{1 \prime} \mid u^{11}(k)\right.\right. \\
& \left.=d^{21}\left(y^{1 \prime}, u^{22}(1), \cdots, u^{22}(k)\right), i=i^{\prime}, \text { for all } k<n\right\}
\end{aligned}
$$

$$
\begin{aligned}
P^{2}\left(n, j^{\prime}\right) & =P_{i}\left\{\left(y^{2 \prime} \mid u^{22}(k)\right.\right. \\
& \left.=d^{12}\left(y^{2 \prime}, u^{11}(1), \cdots, u^{11}(k)\right), j=j^{\prime}, \text { for all } k<n\right\} .
\end{aligned}
$$

Thus, $P^{1}(n, i)$ is the probability, according to DM 2 's personal probability model $P_{j}$, of the possible measurement values $y^{1}$ which would be consistent with DM 1's decisions if DM 1's personal probability model was $P_{i} . P^{2}(n, j)$ can be interpreted similarly. It is easy to see that, for each $i, j \in I, P^{1}(n, i)$, and $P^{2}(n, j)$ are monotone decreasing sequences in $n$. Since the consensus process reaches steady state after a finite number of communications (for $n \geq T$ ), there are three possible outcomes.

1) There exist no $i^{\prime}$ or $j^{\prime}$ such that both

$$
\begin{aligned}
& P^{1}\left(T, i^{\prime}\right) \Pi^{1}\left(i^{\prime}, j\right)>0 \\
& P^{2}\left(T, j^{\prime}\right) \Pi^{2}\left(i, j^{\prime}\right)>0 .
\end{aligned}
$$

2) There exist one $i^{\prime}$ and one $j^{\prime}$ such that

$$
\begin{aligned}
& P^{1}\left(T, i^{\prime}\right) \Pi^{1}\left(i^{\prime}, j\right)>0 \\
& P^{2}\left(T, j^{\prime}\right) \Pi^{2}\left(i, j^{\prime}\right)>0 .
\end{aligned}
$$

3) There exist more than one $i^{\prime}$ or $j^{\prime}$ such that

$$
\begin{aligned}
& P^{1}\left(T, i^{\prime}\right) \Pi^{1}\left(i^{\prime}, j\right)>0 \\
& P^{2}\left(T, j^{\prime}\right) \Pi^{2}\left(i, j^{\prime}\right)>0 .
\end{aligned}
$$

When (35) holds, the inconsistencies among the statistical models $\Pi^{1}$ and $\Pi^{2}$ are detected in the consensus process. It is possible that the true probability model used by DM 1 was not considered possible in DM 2's subjective distribution. In this case, either $d^{12}(T) \neq d^{22}(T)$ or $d^{21}(T) \neq d^{11}(T)$. When (36) holds, either a consensus outcome will be reached for $\omega \in \Omega$, or an inconsistency in the underlying probability models $P_{i}$ and $P_{j}$ will be discovered. If $i=j$, this is the case analyzed in [1]-[5]; in this case, the results of [5] guarantee that $d^{11}(T)=d^{12}(T)=$ $d^{21}(T)=d^{22}(T)$, so a consensus outcome is reached. If $i \neq j$, this is the case analyzed in [6] and [7]. In this case, two types of outcomes are possible: either $d^{12}(T) \neq d^{22}(T)$ or $d^{21}(T) \neq$ $d^{11}(T)$, so that an inconsistency in models $P_{i}$ and $P_{j}$ is detected, or both $d^{11}(T)=d^{21}(T)$ and $d^{22}(T)=d^{12}(T)$. For the second outcome, the results in [6] and [7] imply also that $d^{11}(T)=$ $d^{22}(T)$.

When (37) holds, there is residual ambiguity in both the statistical models and the underlying probability models. As discussed in Example 3, it is possible to have $d^{12}(T)=d^{22}(T)$, $d^{21}(T)=d^{11}(T)$, and $d^{11}(T) \neq d^{22}(T)$ for all $n$, in addition to the other two outcomes. In this case, no inconsistencies have been discovered between either the statistical models $\Pi^{1}$ and $\Pi^{2}$, or the underlying probability models $\boldsymbol{P}_{i}$ and $\boldsymbol{P}_{j}$. Rather, the decision makers have reached a stage where no additional information will be exchanged in the consensus process. At this stage, the DM's agree that a consensus cannot be reached, and discontinue the process.

The above discussion has established the following proposition.

Proposition 7: Assume that the differences between $\Pi^{1}$ and $\Pi^{2}$ are secret knowledge to both decision makers. Then, the consensus process will reach one of three possible outcomes after a finite number of communications.

1) A consensus is reached,

2) DM 1 and DM 2 realize that their underlying statistical models are inconsistent,

3) DM 1 and DM 2 agree to disagree because they cannot gather any additional information from the consensus process.

A result similar to Proposition 7 was obtained in [7]. However, when each DM considers a set of possible underlying probability models for the other DM, as is the case in this section, the consensus process can result in an outcome not predicted by the model of [7]; namely, the DM's can agree to disagree even though their underlying probability models have not been established as inconsistent with each other. This point is illustrated by the following example.

Example 4: Let $(\Omega, F), \mathcal{Y}^{1}, \mathcal{Y}^{2}, X, P_{1}, P_{3}, P_{4}$ be defined as in Examples 2 and 3 . Let $J(\omega, u)$ be defined as in (12), and let $U=$ $[0,1]$. Let $I=\{1,3,4\}$ be the set of possible probability model indexes. Consider the decision rule defined in (7). Assume that $i$ $=1$, and $\Pi^{1}(1,1)=0.3, \Pi^{1}(1,3)=0, \Pi^{1}(1,4)=0.3$, so that DM 1 believes DM 2 is using either model 1 or model 4 with equal probability. Assume $j=4$, and $\Pi^{2}(1,4)=0, \Pi^{2}(3,4)=$ $0.1, \Pi^{2}(4,4)=0.1$, so that DM 2 assumes that DM 1 is using either model 3 or model 4 with equal probability. Assume that $\omega$ $\in a_{1} \cap b_{3}$ and DM communicates first.

The first tentative decision of DM 1 is $u^{\prime \prime}(1)=0.5$. According to DM 2, if DM 1 was using model $P_{3}$, then $u^{21}(1)=0.5$ when $\omega$ $\in a_{1}$, and 0.25 when $\omega \in a_{2}$. If DM 1 was using model $\boldsymbol{P}_{4}$, then $u^{21}(1) \neq 0.25$ for any $\omega$. Hence, DM 2 believes $i=3$, and $\omega \in$ 
$a_{1} \cap b_{3}$. According to his own model, $\boldsymbol{P}_{4}$, DM 2's communication is $u^{22}(1)=0.5$. In communicating $u^{11}(1)=0.5$, DM 1 believes that he has signaled that $i=1$ and $\omega \in a_{1}$. Hence, DM 1 expects $u^{12}(1)=0.5$ if $j=1$ and $\omega \in b_{1}$, or if $j=4$ and $\omega \in b_{3}$. Consequently, DM 1 chooses when the decision makers bring human biases and inaccuracies in probability assessments into the consensus process, a consensus may not be reached even if the decision makers share the same probability model. The results depend explicitly on the Bayesian formulation for incorporating uncertainty concerning the other

$$
\begin{aligned}
u^{11}(2) & =\frac{P_{1}\left(X \mid a_{1} \cap b_{1}\right) P_{1}\left(a_{1} \cap b_{1}\right) \Pi^{1}(1,1)+P_{1}\left(X \mid a_{1} \cap b_{3}\right) P_{1}\left(a_{1} \cap b_{3}\right) \Pi^{1}(1,4)}{\left.P_{1}\left(a_{1} \cap b_{1}\right) \Pi^{1}(1,1)+P_{1}\left(a_{1} \cap b_{3}\right) \Pi^{1}(1,4)\right\}} \\
& =0.375
\end{aligned}
$$

Since models $P_{3}$ and $P_{1}$ have the same distribution conditioned on $a_{1}$, it is also true that $u^{21}(2)=0.375$. Since DM 2 believes he already knows $i=3$, and $\omega \in a_{1} \cap b_{3}$, he learns no additional information, so his decision continues to be $u^{22}(2)=0.5$. This decision conveys no additional information to DM 1 , so the consensus process stalls at this point, and both decision makers agree that a consensus cannot be reached.

\section{CONCLUSION}

In this paper, we have studied the problem of reaching a consensus in a group of decision makers by exchanging tentative decisions using a Bayesian framework. When the decision makers have different probability models and the existence of those differences is secret knowledge, the results of Teneketzis and Varaiya [6], [7] characterized all possible outcomes of the consensus process into two types of outcomes:

1) reaching a consensus decision for the group,

2) reaching a contradiction.

The results of Section III shed additional insight concerning how likely each of these outcomes is. By defining the concepts of a generic outcome and a continuous outcome, we have shown that, when the decision space is continuous-valued and some regularity conditions are met, reaching a contradiction is a generic outcome. In contrast, when the decision space is discrete-valued, both outcomes are continuous, so that small deviations in probability models result in the same outcomes.

One of the limitations of the results of Teneketzis and Varaiya is their assumption that knowledge that probability models could be different is secret knowledge to the decision makers, although in fact the probability models are different. If the decision makers are humans, subject to various biases and inaccuracies in evaluating probabilities [9], knowledge that there can exist differences in probability models is best modeled as common knowledge. In Section IV, we developed a Bayesian framework whereby this knowledge is represented as common knowledge, and the specific individual probability models are represented as private information for each decision maker. In this framework, the consensus process serves both to reveal information concerning the probability model of each decision maker, as well as information concerning the problem uncertainty. A surprising result is that, even when the probability models of the decision makers are identical, and are selected independently from identical probability distributions, there are two possible outcomes.

1) A consensus was reached.

2) A point was reached where both decision makers, on the basis of common information, agree that a consensus cannot be reached.

The second outcome has not been predicted by the previous formulations [1]-[7]. Indeed, it seems to contradict the title of Geanakopoulos and Polemarchakis [4], "We can't disagree forever." Our results in Section IV show that merely admitting the possibility that the probability models are different is sufficient to generate the second outcome. Again, we characterize how likely these outcomes are for both continuous-valued and discretevalued decision spaces $U$.

In conclusion, we have shown that, in our Bayesian framework, decision maker's true probability model. A different formulation, similar to Kreps and Wilson's formulation for sequential games [11], could be developed whereby each decision maker considers only the most likely interpretation of the results as the basis for selecting his tentative decisions. The merits of each formulation rest ultimately in its ability to provide insight into the behavior of humans in consensus decision making.

\section{ACKNOWLEDGMENT}

The authors would like to thank J. R. Simpson for numerous helpful discussions.

\section{REFERENCES}

[1] R. J. Aumann, "Agreeing to disagree," Annals Statist., vol. 4, pp. $1236-1239,1976$.

[2] V. Borkar and P. Varaiya, "Asymptotic agreement in distributed estimation," IEEE Trans. Automat. Contr., vol. AC-27, pp. 650655,1982

[3] J. N. Tsitsiklis and M. Athans, "Convergence and asymptotic agreement in distributed decision problems," IEEE Trans. Automat. Contr., vol. AC-29, pp. 42-50, 1984.

[4] J. D. Geanakopoulos and H. M. Polemarchakis, "We can't disagree forever," J. Economic Theory, vol. 26, pp. 192-200, 1982.

[5] R. B. Washburn and D. Teneketzis, "Asymptotic agreement among communicating decisionmakers," Stochastics, vol. 13, pp. 103-129, 1984.

[6] D. Teneketzis and P. Varaiya, "Consensus in distributed estimation with inconsistent beliefs," Syst. Contr. Lett., vol. 4, pp. 217-221, 1984.

[7] - "Consensus in distributed estimation," in Advances in Signal Processing, V. Poor, Ed., 1986.

[8] J. Harsanyi, "Games with incomplete information played by Bayesian players," Management Sci., vol. 14, pp. 159-182, 1967.

[9] M. H. DeGroot "Researching a consensus," J. Amer. Statist. Association, vol. 69, no. 345, Mar. 1974.

[10] P. Halmos, Measure Theory. New York: Springer-Verlag, 1970.

[11] D. M. Kreps and R. Wilson, "Sequential equilibria," Econometrica, vol. 50,1982 .

[12] P. Levine and J. P. Ponssard, "The values of information in some nonzero sum games," Int. J. Game Theory, vol. 6, no. 4.

David A. Castañon (S'68-M'79) for a photograph and biography, see p. 49 of the January 1988 issue of this TRANSACTIONS.

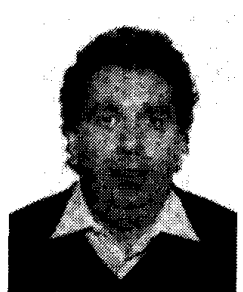

Demosthenis Teneketzis received the B.S. degree in electrical engineering from the University of Patras, Patras, Greece, in 1974, and the M.S. E.E., and Ph.D. degrees in electrical engineering from Massachusetts Institute of Technology, Cambridge, in 1976, 1977, and 1979, respectively.

From 1979 to 1980 he worked for System Control Inc., Palo Alto, CA, and from 1980 to 1984 he was with Alphatech Inc., Burlington, MA. Since September 1984 he has been with the University of Michigan, Ann Arbor, where he is presently an Associate Professor of Electrical Engineering and Computer Science. His current research interests include stochastic systems and control, team theory, game theory, decentralized systems, information theory, and queueing networks. 